\title{
In-Plane Sensitive Magnetoresistors as a Hall Device ${ }^{+}$
}

\author{
Siya Lozanova, Ivan Kolev, Avgust Ivanov and Chavdar Roumenin * \\ Institute of Robotics at Bulgarian Academy of Sciences, 1113 Sofia, Bulgaria; lozanovasi@abv.bg (S.L.); \\ ivankolev91@gmail.com (I.K.); avgust.ivanov@abv.bg (A.I.) \\ * Correspondence: roumenin@bas.bg; Tel.: (+359-2)-870-3361 \\ † Presented at the Eurosensors 2018 Conference, Graz, Austria, 9-12 September 2018. \\ Published: 5 December 2018
}

\begin{abstract}
A novel coupling of a pair of identical two-contact (2C) magnetoresistors transformed into an in-plane sensitive Hall device is presented. The ohmic contacts are cross-linked, also adding a load resistor bridge, providing for constant current mode of operation and eliminating the inevitable parasitic offset. This silicon configuration, apart from its simplified layout, has linear and odd output voltage as a function of the magnetic field and current. The quadratic and even magnetoresistance in the two parts of this innovative device is completely compensated, which ensures high measurement accuracy alongside with identification of the magnetic field polarity. The experimental prototypes feature sensitivity of $110 \mathrm{~V} / \mathrm{AT}$. The mean lowest detected magnetic induction $B$ at supply current of $3 \mathrm{~mA}$ over frequency range $f \leq 100 \mathrm{~Hz}$ at a signal-to-noise ratio equal to unity is $B \min \approx 10 \mu \mathrm{T}$. The high performance and the complete electrical, temperature and technological matching of the parts of this unusual Hall device make it very promising for many practicalapplications.
\end{abstract}

Keywords: in-plane sensitive magnetic-field configurations; magnetoresistors; silicon Hall effect device

\section{Introduction}

The well-known 4C in-plane sensitive Hall devices (HD) are widely used in numerous configurations as universal sensors for practical application and last, but not least - to compensate the main disadvantage in differential output transducers - the offset, frequently through dynamic cancellation techniques. In these architectures, one outer and one inner contact are the supply and the other two contacts are the output [1-9]. By switching the input and output electrodes of the Hall plate and summing-up algebraically the two obtained voltages, the offset can be significantly decreased $[5,7,8]$. These sensors, however, feature some drawbacks, such as: different values of the individual Hall potentials, generated on the two output terminals in a homogeneous magnetic field, output non-linearity, and offset. All these disadvantages result from the low inherent layout symmetry with asymmetry of the current lines with respect to the output terminals. Therefore, non-uniform Lorentz force action in different sections of the current paths is available. The described defects reduce the measurement accuracy. In this paper, a novel in-plane symmetrical HD based on a two magnetoresistor structures overcoming the mentioned disadvantages is presented.

\section{Sensor Design and Operation Principle}

The new configuration contains two identical $n$-Si regions established in parallel to one another. On the top surfaces, two ohmic contacts are formed $-C_{1}, C_{2}$ and $C_{3}, C_{4}$, respectively, Figure 1. A deep 
surrounding $p$-ring is also implemented, reducing the surface current's spreading and confines the transducer region into the substrate bulk.

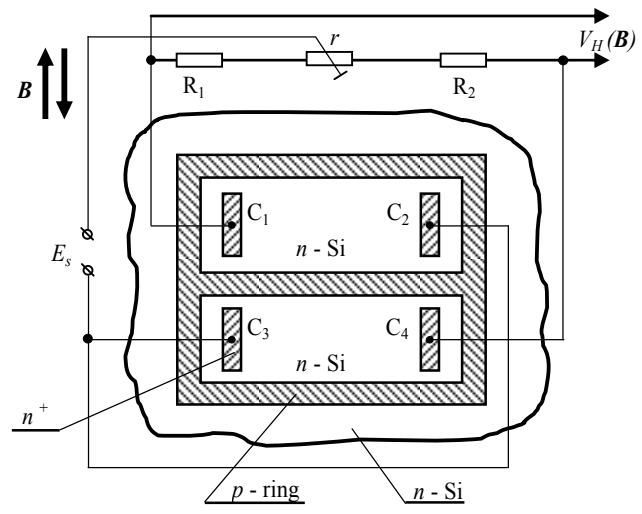

Figure 1. Schematic top-view of two identical $n$-Si 2C magnetoresistors on the same chip forming in-plane sensitive Hall device. The original coupling transforms the magnetoresistors into a symmetrical 4C Hall element.

The magnetic field $B$ is parallel to the long sides of the contacts. The pair of terminals are cross-coupled, $C_{2}-C_{3}$, whereas, between $C_{1}$ and $C_{4}$, high-impedance equal in value resistors $R_{1}=R_{2}$ and a low-impedance trimmer $r$ are connected. The inevitable offset $V_{\mathrm{H}}(0) \neq 0$ is fully compensated by trimmer $r$. The experimental prototype has been implemented using part of the processing steps applied in bipolar IC technology. The carrier's concentration in the $n$-Si substrate is $n \sim 4.3 \times 10^{15} \mathrm{~cm}^{-3}$. Similarly to [9], four masks are employed. The size of contacts $C_{1} \ldots C_{4}$ is $50 \times 10 \mu \mathrm{m}^{2}$, and the width of the $p$-ring on the chip surface is about $20 \mu \mathrm{m}$. The penetration of the current trajectory into the $n$-Si substrate reaches a depth of $30-40 \mu \mathrm{m}$. The novel in-plane sensitive Hall device is in hybrid realization (resistor elements $R_{1}, R_{2}$ and $r$ are discrete).

Components $I_{\mathrm{c} 1,2}$ and $I_{\mathrm{c} 4,3}$ flow in the substrate when the supply Esis switched. The planar ohmic contacts $C_{1} \ldots C_{4}$ represent equipotential planes, to which, in the absence of external magnetic field $B$, $B=0$, currents $I_{c_{1}}, I_{\mathrm{C}_{2}}$, and $I_{\mathrm{c}_{3},} I_{\mathrm{c} 4}$ respectively flow perpendicularly to the upper surfaces of the slabs, deeply penetrating into the bulk. The current lines $I_{\mathrm{c} 1,2}$ and $I_{\mathrm{C}_{4}, 3}$ in the other parts of the two substrates are parallel to their upper surfaces. Therefore, the two trajectories $I_{\mathrm{c} 1,2}$ and $I_{\mathrm{c} 4,3}$ are curvilinear. As a result of the uniformity of the two substrates, as well as of contacts $C_{1} \ldots C_{4}$, the two currents $I_{c 1,2}$ and $-I_{C 4,3}$ are equal in magnitude and opposite in sign. If, as a result of technological imperfections, mechanical strain and stress during chip capsulation, temperature gradients and the like [4-6], at output $V_{\mathrm{H}}(\boldsymbol{B})$ of the device, at field $B=0$, offset $V_{\mathrm{H}}(B=0) \neq 0$ appears, notwithstanding that load resistors $R_{1}$ and $R_{2}$ are equal, $R_{1}=R_{2}$. Varying the value of the low ohmic trimmer $r$, full compensation of the negative offset at differential output is achieved, $V_{\mathrm{H}}(B=0)=0$. The resistors $\mathrm{R}_{1}$ and $\mathrm{R}_{2}$ are at least by one order greater than the effective resistance between the ohmic contacts $C_{1} \ldots C_{4}$.

The application of the measured magnetic field $B$ in parallel to the long sides of contacts $C_{1} \ldots C_{4}$, Figure 1, results in deformation of current lines $I_{\mathrm{C} 1,2}$ and $-I_{\mathrm{C} 4,3}$ along the entire length of the non-linear trajectories, i.e. the electric symmetry of the trajectories is disturbed. This is due to the action of Lorentz force $F_{\mathrm{L}}, F_{\mathrm{L}}=q V_{\mathrm{dr}} \times \boldsymbol{B}$, where $q$ is the electron's elementary charge, and $V_{\mathrm{dr}}$ is the vector of the mean drift velocity of the electrons in the substrates. Therefore, as a result of Lorentz deflection, depending on the specific directions of currents $I_{\mathrm{c} 1,2}$ and $-I_{\mathrm{C} 4,3}$ and of the magnetic field \pm $B$, the trajectories shrink and expand, respectively. For this reason, in the bulks and on the surfaces with planar contacts $C_{1} \ldots C_{4}$, two effects are generated simultaneously. One of them is the quadratic even magnetoresistance $\mathrm{MR} \sim B^{2}$, resulting in increase of the length of the trajectories of the current $[4,6]$. The other sensor mechanism is the linear and odd Hall effect $V_{\mathrm{H}} \sim \pm B$, resulting from the additional non-equilibrium charges on the surfaces with contacts $C_{1}$ and $C_{2}$, and $C_{3}$ and $C_{4}$, respectively. A key specific of the new solution is that the two substrates interact functionally and constitute an integrated sensor system, notwithstanding that the transducer zones are separated from one another. 
The chosen original cross-coupling of the two pairs of magnetoresistors and the differential output $V_{\mathrm{H}}$ formed by the two load resistors $\mathrm{R}_{1}$ and $\mathrm{R}_{2}$ accomplishes synphase suppressing (compensation) of the parasitic quadratic voltage MR $\sim B^{2}$. Thus, the linear and odd Hall voltage $V_{\mathrm{H}} \sim$ $\pm B$ is the output signal of the new in-plane sensitive Hall device.

The output generates information simultaneously for the value of induction $B$ and the direction (sign) of the magnetic vector $\pm \boldsymbol{B}$. The linear output Hall voltage $V_{\mathrm{C} 1,4}(\boldsymbol{B}) \equiv V_{\mathrm{H}}(\boldsymbol{B})$ in Figure 1 increases significantly the metrological accuracy of the device.

\section{Results}

The output characteristics $V_{\mathrm{H}}(\boldsymbol{B})$ of the Hall configuration are presented in Figure 2a. The linear and odd output $V_{\mathrm{H}}(\boldsymbol{B})$ identifies the magnetic field polarity $\pm \boldsymbol{B}$. The non-linearity does not exceed $0.5 \%$ within the range $+0.6 \mathrm{~T} \div-0.6 \mathrm{~T}$, the sensitivity is $S_{\mathrm{R}} \approx 110 \mathrm{~V} / \mathrm{AT}$. The individual sensitivities on the two contacts $\mathrm{C}_{1}$ and $\mathrm{C}_{4}$ of the $\mathrm{HD}$ assessed by potentials $V_{\mathrm{C}}(\boldsymbol{B})$ and $V_{\mathrm{C} 4}(\boldsymbol{B})$, respectively, are equal, $\mathrm{S}_{\mathrm{RI}} \approx 55 \mathrm{~V} / \mathrm{AT}$, Figure $2 \mathrm{~b}$. The quadratic and even magnetoresistance $\mathrm{MR} \sim B^{2}$ on the two terminals $\mathrm{C}_{1}$ and $\mathrm{C}_{4}$ is fully compensated.

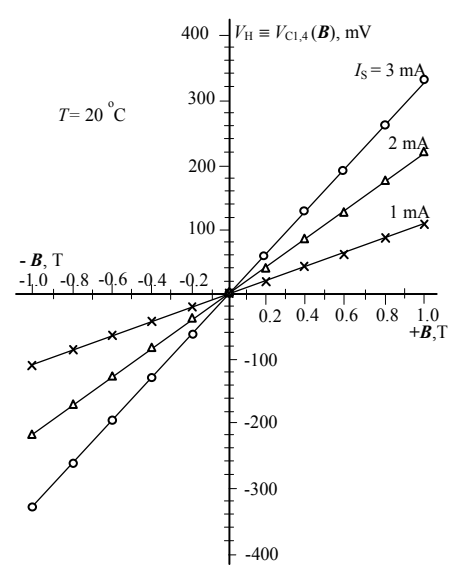

(a)

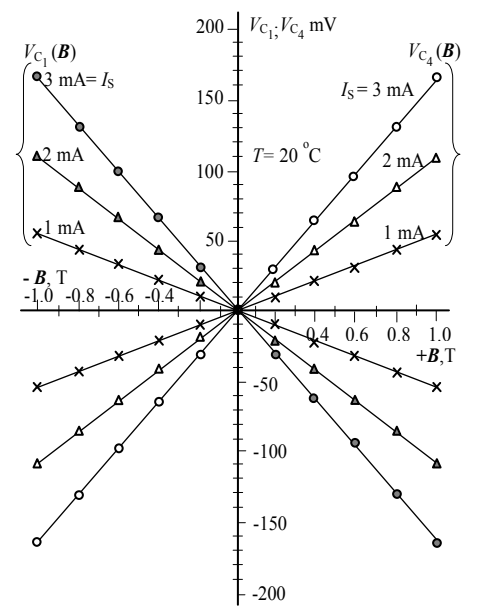

(b)

Figure 2. (a) Output characteristics $V_{\mathrm{C} 1,4}(\boldsymbol{B}) \equiv V_{\mathrm{H}}(\boldsymbol{B})$ of the new HD, the offset is compensated in advance. The obtained magnetosensitivity is about $\mathrm{S}_{\mathrm{RI}} \approx 110 \mathrm{~V} / \mathrm{AT}$ at temperature $T=20{ }^{\circ} \mathrm{C}$; (b) Individual sensitivities on the two contacts $\mathrm{C}_{1}$ and $\mathrm{C}_{4}$ of the $\mathrm{HD}$ assessed by potentials $V_{\mathrm{C} 1}(\boldsymbol{B})$ and $V_{\mathrm{C} 4}(\boldsymbol{B})$, respectively are equal, $\mathrm{S}_{\mathrm{RI}} \approx 55 \mathrm{~V} / \mathrm{AT}$.

The measured power spectral density of the internal noise $V_{\mathrm{Cl}, 4}(0) \equiv \mathrm{V}_{\mathrm{H}}(0)$ is shown in Figure 3, the supply current $I_{\mathrm{s}}$ is a parameter, $T=20^{\circ} \mathrm{C}$. The mean lowest detected magnetic induction $B$ at supply current of $3 \mathrm{~mA}$ over a frequency range $f \leq 500 \mathrm{~Hz}$ at a signal-to-noise ratio equal to unity is $B_{\min } \approx 10 \mu \mathrm{T}$.

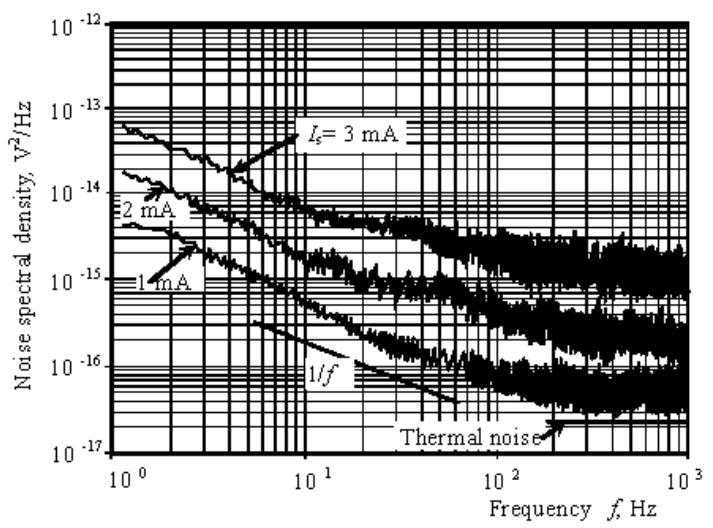

Figure 3. Spectral density of internal noise $V_{\mathrm{C} 1,4}(0)$, the supply current $I_{\mathrm{s}}$ is as a parameter, $T=20^{\circ} \mathrm{C}$. 


\section{Conclusions}

The innovation of the arrangement and the original connection with one current source accomplish a magnetosensitive device with new properties-linear and odd voltage is generated at its differential output. For the first time, using two separate non-linear magnetoresistors, a linear Hall sensor is realized. The universality of the proposed HD and the simplified technological realization, as well as the promising results shows that it may be suitable for many industrial applications.

Acknowledgments. This work was supported by Sci. Res. Fund under project No. DN 07/18-15.12.2016

\section{References}

1. Roumenin, C.S. Hall Effect Sensor, Bulg. Patent No. 41974/06.05.1986, 1986.

2. Roumenin, C.S. Parallel-field Hall microsensor. Compt. Rendus ABS 1987, 40, 59-62.

3. Falk, U. A symmetrical vertical Hall-effect device. Sens. Actuators A 1990, 21-23, 751-753.

4. Roumenin, C. Solid State Magnetic Sensors, Elsevier, 1994; Microsensors for magnetic field. In MEMS - A Practical Guide to Design, Analysis and Application; Korvink, J.G., Paul, O., Eds.; William Andrew Publisher: Norwich, NY, USA, 2006, pp. 453-521.

5. Kaufmann, T. On the Offset and Sensitivity of CMOS-Based Five-Contact Vertical Hall Devices; Der Andere Verlag: Tonning, Germany, 2013; Volume 21, p. 147.

6. Lozanova, S.V.; Roumenin, C.S. Parallel-field silicon Hall effect microsensors with minimal design complexity. IEEE Sens. J. 2009, 9, 761-766.

7. Schurig, E.; Schott, C.; Besse, P.-A.; Popovic, R.S. CMOS integrated vertical Hall sensor with low offset. In Proceedings of the XVI European Conference on Solid-State Transducers, Prague, Czech Republic, 15-18 September 2002, pp. 868-871.

8. Schurig, E.; Schott, C.; Besse, P.-A.; Demierre, M.; Popovic, R.S. $0.2 \mathrm{mT}$ residual offset of CMOS integrated vertical Hall sensors. Sens. Actuators A 2004, 110, 98-104.

9. Schott, C.; Popovic, R. Integrated 3-D Hall magnetic field sensor. In Proceedings of the Transducers '99, Sendai, Japan, 7-10 June 1999; Volume 1, pp. 168-171.

(C) 2018 by the authors. Licensee MDPI, Basel, Switzerland. This article is an open access article distributed under the terms and conditions of the Creative Commons Attribution (CC BY) license (http://creativecommons.org/licenses/by/4.0/). 